\title{
DDEC: Dragon database of genes implicated in esophageal cancer Magbubah Essack ${ }^{1}$, Aleksandar Radovanovic ${ }^{1}$, Ulf Schaefer ${ }^{1}$, Sebastian Schmeier ${ }^{1}$, Sundararajan V Seshadri ${ }^{1}$, Alan Christoffels ${ }^{1}$, Mandeep Kaur ${ }^{1}$ and Vladimir B Bajic*1,2
}

\author{
Address: ${ }^{1}$ South African National Bioinformatics Institute, University of the Western Cape, Bellville, South Africa and ${ }^{2}$ Computational Bioscience \\ Research Center (CBRC), Mathematical and Computer Science and Engineering Division, King Abdullah University of Science and Technology \\ (KAUST), Thuwal, Saudi Arabia \\ Email: Magbubah Essack - magbubah@sanbi.ac.za; Aleksandar Radovanovic - aleksandar@sanbi.ac.za; Ulf Schaefer - ulf@sanbi.ac.za; \\ Sebastian Schmeier - sebastian@sanbi.ac.za; Sundararajan V Seshadri - sundar@sanbi.ac.za; Alan Christoffels - alan@sanbi.ac.za; \\ Mandeep Kaur - mandeep@sanbi.ac.za; Vladimir B Bajic* - vladimir.bajic@kaust.edu.sa \\ * Corresponding author
}

Published: 6 July 2009

BMC Cancer 2009, 9:219 doi:10.1 186/147|-2407-9-219
Received: 12 December 2008

Accepted: 6 July 2009

This article is available from: http://www.biomedcentral.com//47/-2407/9/219

(C) 2009 Essack et al; licensee BioMed Central Ltd.

This is an Open Access article distributed under the terms of the Creative Commons Attribution License (http://creativecommons.org/licenses/by/2.0), which permits unrestricted use, distribution, and reproduction in any medium, provided the original work is properly cited.

\begin{abstract}
Background: Esophageal cancer ranks eighth in order of cancer occurrence. Its lethality primarily stems from inability to detect the disease during the early organ-confined stage and the lack of effective therapies for advanced-stage disease. Moreover, the understanding of molecular processes involved in esophageal cancer is not complete, hampering the development of efficient diagnostics and therapy. Efforts made by the scientific community to improve the survival rate of esophageal cancer have resulted in a wealth of scattered information that is difficult to find and not easily amendable to data-mining. To reduce this gap and to complement available cancer related bioinformatic resources, we have developed a comprehensive database (Dragon Database of Genes Implicated in Esophageal Cancer) with esophageal cancer related information, as an integrated knowledge database aimed at representing a gateway to esophageal cancer related data.

Description: Manually curated 529 genes differentially expressed in EC are contained in the database. We extracted and analyzed the promoter regions of these genes and complemented gene-related information with transcription factors that potentially control them. We further, precompiled text-mined and data-mined reports about each of these genes to allow for easy exploration of information about associations of EC-implicated genes with other human genes and proteins, metabolites and enzymes, toxins, chemicals with pharmacological effects, disease concepts and human anatomy. The resulting database, DDEC, has a useful feature to display potential associations that are rarely reported and thus difficult to identify. Moreover, DDEC enables inspection of potentially new 'association hypotheses' generated based on the precompiled reports.
\end{abstract}

Conclusion: We hope that this resource will serve as a useful complement to the existing public resources and as a good starting point for researchers and physicians interested in EC genetics. DDEC is freely accessible to academic and non-profit users at http://apps.sanbi.ac.za/ddec/. DDEC will be updated twice a year. 


\section{Background}

The major histological form of esophageal cancer (EC), esophageal squamous cell carcinoma (ESCC), comprises $90 \%$ of ECs worldwide $[1,2]$. The poor prognosis of EC results in a five year survival rate of $5-20 \%$ [3]. The lethality of EC stems from our inability to detect the disease during the early stage, combined with the lack of effective therapies for advanced-stage disease. Like most diseases, EC arises as a consequence of errors occurring in the cellular regulatory system or errors being introduced into the genome as mutations causing cellular behavior to deviate from the norm [4]. Identifying the mechanisms by which the genomic information is controlled in EC will provide further insights into partially understood cellular and molecular functioning that characterizes this disease.

Gene expression in EC is a multifunctional process influenced by chromatin remodeling and the interplay between transcription regulatory proteins and DNA sequences known as transcription factor binding sites (TFBSs) $[5,6]$. This combination of transcription regulatory proteins, TFBSs, and affected transcripts, defines the transcription regulatory networks (TRNs) that are responsible for the regulation of every transcript encoded in the genome. Knowledge of these transcripts and the control mechanisms of their initiation set the stage for inferring transcriptional regulatory networks and may help in search for the therapeutic mechanisms to potentially correct or compensate for the errors underlying pathological states of EC.

Efforts made by the scientific community to improve the survival rate associated with EC have resulted in a wealth of scattered research data. Researchers need to sieve through this scattered research data to identify relevant research findings. However, this phase hampers the research process as the compiling of the relevant information is tedious and time consuming. In an attempt to enhance research endeavors related to EC we have developed Dragon Database of Genes Implicated in Esophageal Cancer (DDEC) as an integrated knowledge database that contains information about various genes differentially expressed in EC. It should be noted that there are two initiatives aimed at coordinating activities in producing resources related to cancer research, such as the International Cancer Genome Consortium - ICGC http:// www.icgc.org/ and caBIG (cancer Biomedical Informatics Grid $^{\mathrm{TM}}$, http://cabig.cancer.gov/. These two intend to promote specific data formats and other conditions that will enable easier integration of cancer-related resources. There are cancer related databases that include information on EC, such as Cancer Gene Expression Database (CGED) [7], PDQ [8] and Oncomine [9]. CGED houses a collection of gene expression and clinical data from a large number of patients with major cancers including EC.
CGED expression data have been obtained by adaptortagged competitive PCR (ATAC-PCR) and allows researchers to explore the correlation between gene expression and clinical data for future diagnostic application [7]. PDQ is the National Cancer Institute's (NCI's) cancer database that includes peer-reviewed summaries on cancer treatment, screening, prevention, genetics, and complementary and alternative medicine [8]. The Oncomine initiative collects and analyzes all published cancer microarray data and currently house EC-related microarray data [9]. However, none of the current public databases focuses on genes implicated in EC and their potential associations with other relevant biological, biochemical and medical entities. Moreover, DDEC provides a combination of features for exploration of information related to EC-implicated genes that cannot be found elsewhere, such as filtering for putative transcription factors shared amongst promoters of EC-implicated genes, inference of association networks and precompiled reports that provide insights into other human genes and proteins, metabolites and enzymes, toxins, chemicals with pharmacological effects, disease concepts and human anatomy associated with differentially expressed EC-implicated genes. It also enables finding rare information that will be likely missed in the common literature search. As a special feature, DDEC provides a module for generation of 'association hypotheses' between concepts related to EC-implicated genes. Batch queries and database dump are also provided. We thus believe that DDEC represents a useful complement to the existing databases and will contribute to more efficient EC-related research. DDEC is freely accessible for academic and non-profit users at http:// apps.sanbi.ac.za/ddec/. The semi-automated methodology used to populate DDEC genes and related data will be used to update the database twice a year.

\section{Construction and content}

The DDEC is based on the three-tier (layer) (data, logic and presentation) architecture (Figure 1). The presentation layer is web-based and implemented in DHTML and Javascript. The logic layer was implemented as a number of server side PHP and Perl modules interfaced with the data layer. Data layer is MySQL, and for the text-mining purposes, file system based. The relational database design strictly distinguishes between tables that contain data entities and tables that establish logical connections between these data entities. The central data entity is the gene, to which most other data entities are linked. Other important data entities are transcription related such as transcription start sites (TSSs) and transcription factors (TFs). This is reflected in the entry points that a user can chose between on the top level of the web-interface.

Information in the DDEC is structured into four distinct parts: 


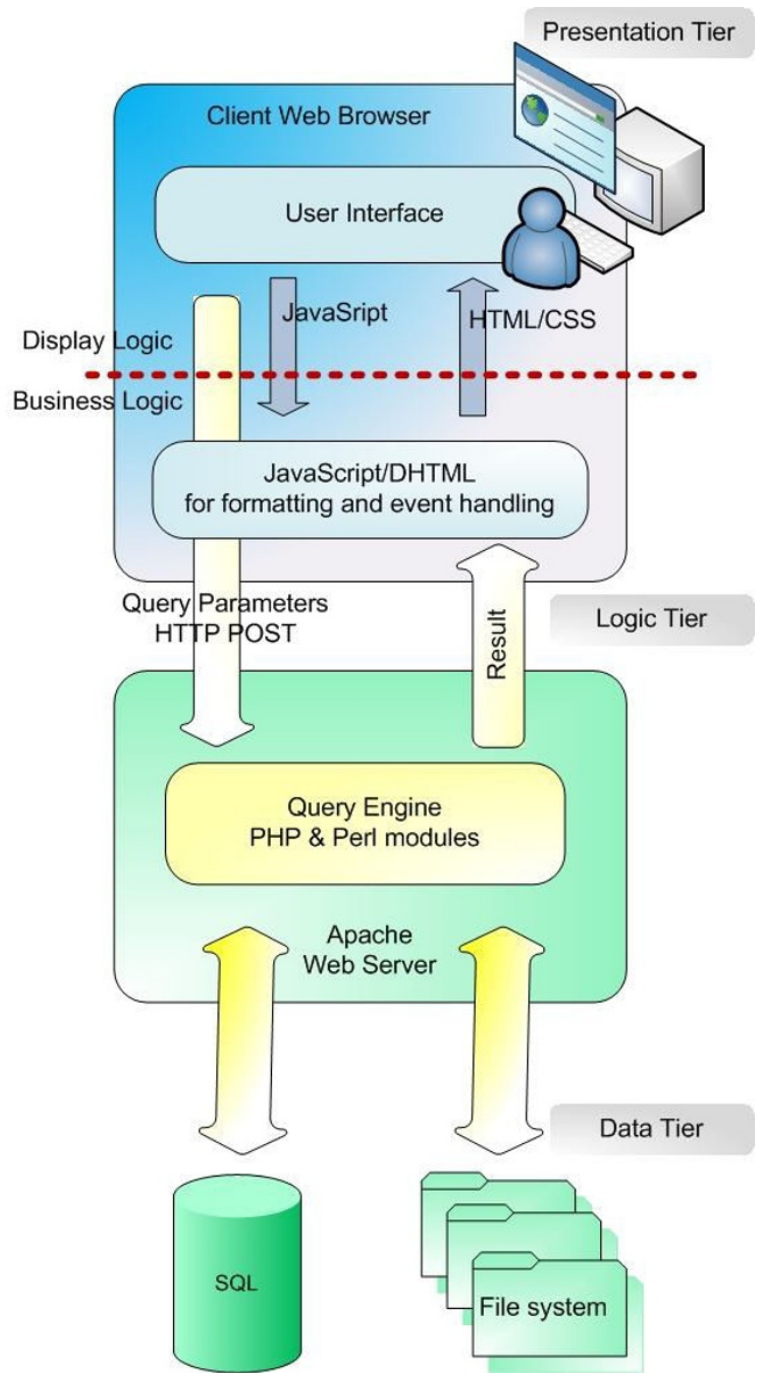

\section{Figure I}

The schematic representation of the DDEC structure. The DDEC is based on the three-tier (layer) architecture, namely; data, logic, and presentation.

(I) Platform that can be used to search the integrated gene information through standardized vocabularies.

(II) Selection of the genes of interest from the list. This search criteria provides users with gene details such as; general information, gene in other resources, experimental evidence, related proteins, associated pathways, associated diseases, orthologous genes, regulations and text-mined reports that can support building interactive association networks.

(III) Transcription regulation information which includes all putative TFBSs for the EC-implicated genes in DDEC. This segment is useful for gene regula- tion studies since TFBSs of interest can be selected and the results will list each TFBS and gene promoter with corresponding TFBSs. Genes sharing all the selected TFBSs are listed as well.

(IV) Batch queries and data download interface is provided to increase utility for users.

DDEC contains information on EC-implicated genes compiled based on scientific publications from PubMed. The PubMed database was queried with keyword expression: "esophageal (cancer OR cancers OR tumor OR tumors OR carcino* OR adenocarc* OR malign* OR neoplasm*)" on 31/01/2008 and 35,892 PubMed abstracts were retrieved. The search for relevant publications was further refined using the licensed Dragon Exploration System (DES) from OrionCell http://www.orioncell.org, that has an integrated Biomedical Text-Miner tool. DES retrieved a list of 1677 putative genes associated with EC from the extracted abstracts. Biologists then evaluated information about experimental conditions these genes have been subjected to using full-text articles whenever possible, and abstracts in other cases. When the available information was insufficient to deduce the correct experimental conditions, the gene has been discarded. Taking into account that experimental conditions influence gene expression, DDEC provide details of the cell line, tissue or cell type, expression status, disease stage, tumor grade, esophageal cancer type and laboratory method reported in literature.

A final list of 529 genes was identified in this way and used to populate the database. The general information about the genes, which include HGNC ID, approved symbol, approved name, entrez ID, previous symbol, previous name, aliases, OMIM-related information, and chromosome location, were extracted from sources such as HUGO [10]http://www.genenames.org/ and GeneCards [11] http://www.genecards.org/index.shtml. Included in the database are gene related identifiers such as EMBL [12] http://www.ebi.ac.uk/embl/, Ensembl [13]http:// www.ensembl.org/index.html, Refseq [14], Genbank [15]http://www.ncbi.nlm.nih.gov/, Unigene [16]http:www.ncbi.nlm.nih.gov/sites/entrez?db=uni gene\&orig db, Uniprot [17]http://www.ebi.ac.uk/uni prot/, Swiss-Prot [18] http://www.expasy.ch/sprot/ and PDB [19] http://www.rcsb.org/pdb/home/home.do. ID conversion tools like IDconvertor [20] http://idcon verter.bioinfo.cnio.es/ and Onto-tools [21] http://vor tex.cs.wayne.edu/ontoexpress/servlet/UserInfo were used to convert between different types of identifiers. A summary of the statistics of the above mentioned features are listed in documentation. We have provided links to the relevant sources of data such as gene ontologies [22] http:/ /www.geneontology.org/, Evoc [23] http://www.evocon 
tology.org $/$, and Reactome pathway data [24] $\underline{\text { http:// }}$ www.reactome.org/.

As a useful feature, we generated lists of putative TFBSs that map to the promoter regions of EC-implicated genes allowing users to identify genes that share common TFBSs. For this purpose, promoter sequences were extracted using mainly FANTOM3 CAGE tag data [25], as well as TOUCAN v. 3.0.2 [26]. To map TFBSs to promoters we used the TRANSFAC Professional database v.11.4 [27]. All TRANSFAC mammalian matrix models of binding sites [28]were mapped using the $\mathrm{Match}^{\mathrm{TM}}$ program with minFP profiles for optimized thresholds of the matrix models [29]. The complete list of 529 genes was used to extract promoter sequences for the identification of putative TFBSs. Promoter sequences of 409 genes (1200 bp upstream and 200 bp downstream from the transcription start site, TSS) were extracted from the Fantom3 CAGE tag data that correspond to 1582 transcription start sites (TSSs) that each has at least five tags in the tag cluster and a minimum of three tags in the representative tag [25]. An additional 108 promoter sequences (1200 bp upstream and $200 \mathrm{bp}$ downstream from the TSS) were extracted using Toucan v. 3.0.2 [26].

As an additional feature, for each of the 529 EC-implicated genes, we extracted all related PubMed documents and analyzed them using DES. DES uses a dictionary based text-mining approach to extract information used for the precompiled reports by mapping the entities from the dictionaries to the submitted PubMed documents. We applied six manually curated DES dictionaries namely; human genes and proteins, metabolites and enzymes, toxins, chemicals with pharmacological effects, disease concepts and human anatomy. These dictionaries were compiled from literature and public databases. The accuracy of this integrated data has been evaluated in Sagar $e t$ $a l$. in terms of precision, recall and F-measure. The analysis of the results displayed precision and recall ranging from $81 \%-100 \%$ and with an average F-measure of $92.9 \%$ for the SCN1A gene [30]. The precompiled reports in this study are incorporated in the DDEC and provide the user with a possibility to inspect possible interactions associated with the genes of interest and associated networks of relevant biomedical entities. An additional feature in DES allows for hypotheses to be generated between two dictionary entries that are linked to a common dictionary entry. This tool allows the user to test the hypotheses generated by retrieving PubMed documents related to the two dictionary terms linked through the hypothesis, if no PubMed documents are retrieved the hypothesis may warrant further exploration. This functioning of the text-mining modules of DDEC is based on similar concepts as used in Pan et al. [31] and Bajic et al.
[32]. DES has also been employed in the creation of a module for the ovarian cancer database, DDOC [33].

Batch queries and data download are provided to increase utility for users. Further, a database dump has been provided to support integration with other database resources.

The above outlined process of biocurated data collection and integration will be repeated twice yearly as an update process. Updates will incorporate extracting abstracts from the last update day to current day. This semi-automated process is more time consuming than current automated update systems but has the advantage of reducing redundant information.

\section{Utility and discussion}

DDEC provides a comprehensive compilation of information obtained from published EC research, complemented with the information from public databases and information derived from computational analysis. The information captured in DDEC is centered on genes differentially expressed in EC. The information used for selection of genes to be included in DDEC was curated by biologists. Only genes that satisfy all conditions listed below are included in DDEC:

(i) Genes that are differentially expressed in human EC with experimental proof.

(ii) Differential expression of EC-implicated genes has not been influenced by anti-cancer therapy.

(iii) Differentially expressed EC-implicated genes have not been artificially constructed.

Microarray data has been excluded at this stage as the results obtained using high throughput technologies are debatable in terms of deciding about a meaningful level of gene expression and statistical methods used for analysis and interpretation of data $[34,35]$. However, as a future prospect we will expand the database by adding a subset for raw expression data and analysis of the EC-related microarray data.

DDEC contains precompiled text-mined and data-mined reports that allow for easy exploration of information about associations of EC-implicated genes with other genes and proteins, metabolites and enzymes, toxins, chemicals with pharmacological effects, disease concepts, human anatomy, pathways and pathway reactions. Moreover, DDEC provides for potentially new 'association hypotheses' generated in the precompiled reports. It also provides frequency of associations that allows users to observe rare associations with the genes of interest that 
will usually be overlooked in a normal literature search taking into account the huge volume of data available. DDEC can be used to answer questions such as:

(1) Is my gene of interest differentially expressed in EC, i.e. is it an EC-implicated gene as defined here?

(2) Which putative transcription factors regulate the expression of an EC-implicated gene or sets of these genes?

(3) Which of the other EC-implicated genes in DDEC are regulated by the same transcription factor (or factors) as the gene of interest?

(4) My gene of interest has putative associations with other biomedical concepts. What are these concepts and what are the documents from which such associations are deduced so that I can explore them?

The potential uses and advantages of the database are described in the documentation section http:// apps.sanbi.ac.za/ddec/ddec.pdf. An example of data analysis has been included in the documentation and should help users to understand and utilize different functions implemented in this database to maximize information exploration and extraction.

Kaur et al. recently published DDOC, an ovarian cancer (OC) database housing 379 OC-related genes using the same database model and query interface [33]. To explore whether the EC and OC database content characterize functionally distinct groups of genes, the categories where probed for statistical over-representation of GO terms $[22,36]$. For this analysis we compared the EC and OC gene lists. We found 123 genes to be common to both cancer types while 406 genes were unique to EC and 256 genes were unique to OC. Generally, all categories were characterized by the majority of genes forming part of the broad terms, apoptosis and cell cycle. However, these categories were primarily over-represented for the genes common to both EC and OC (see Table 1). The gene list unique to EC was found to be enriched in functionally distinct groups such as 'neuron differentiation and development' and 'epidermis development' while the gene list unique to $\mathrm{OC}$ was found to be enriched in functionally distinct groups such as 'sex differentiation and development' and 'embryonic development' (see Table 1).

We further identified which KEGG pathways (see additional file 1) are enriched for the genes unique to EC, genes unique to $\mathrm{OC}$ and the genes common to $\mathrm{EC}$ and $\mathrm{OC}$ [37]. We found the MAPK signaling pathway, ErbB signaling pathway and p53 signaling pathway to be most pronounced pathways for genes common to EC and OC. The pathways most pronounced for the genes unique to EC were the MAPK signaling pathway, Wnt signaling pathway, with androgen and estrogen metabolism being unique to this group. The MAPK signaling pathway, ErbB signaling pathway and TGF-beta signaling pathways were most pronounced for the genes unique to OC.

Above analysis suggests that distinct categories of genes participating in specific pathways are involved in pathogenesis of different types of cancers. These cancer specific categories of genes can be investigated as potential biomarkers for prognosis and diagnosis of the disease.

In future, we intend to incorporate the effect of current therapeutic drugs. Additional features that may enhance

Table I: A comparison of the DDEC and DDOC gene lists.

\begin{tabular}{lccc}
\hline $\begin{array}{l}\text { Gene Ontology terms representing } \\
\text { functionally distinct groups }\end{array}$ & $\begin{array}{c}\text { Genes unique for Esophageal } \\
\text { Cancer (EC) }\end{array}$ & $\begin{array}{c}\text { Gene unique for Ovarian Cancer } \\
(\mathrm{OC})\end{array}$ & $\begin{array}{c}\text { Genes common to EC and OC } \\
\begin{array}{l}\text { Neuron differentiation and } \\
\text { development }\end{array}\end{array}$ \\
\hline Epidermis development & 5.03 & 1.06 & 0 \\
\hline Sex differentiation and development & 0.36 & 1.36 & 1.66 \\
\hline Embryonic development & 0.76 & 1.85 & 0 \\
\hline Regulation of apoptosis & 8.09 & 9.84 & 13.22 \\
\hline Regulation of cell cycle & 11.99 & 11.31 & 14.73 \\
\hline
\end{tabular}

* The values tabulated in Table I represent the overall enrichment score for the group based on EASE scores of each term members. The higher the score the more enriched.

A comparison of Esophageal and Ovarian Cancer genes by characterizing functionally distinct groups based on Gene Ontology terms. 
search and retrieval of DDEC information will be added in due course, as well as incorporation of DDEC into ICGC, caBIG and LinkOut. DDEC will further be updated twice a year and will continue to grow in both content and functionality.

\section{Conclusion}

DDEC is an integrated knowledge database aimed at representing a gateway to EC-related data. DDEC houses information associated with 529 hand-curated human genes implicated in EC and allows the users to easily access the wealth of EC related data that is typically difficult to find and not easily amendable to data mining. Users are also provided with the DES interface that allows for the easy exploration of information, viewing of potential associations that are rarely reported and thus difficult to identify and inspection of potentially new 'association hypotheses' generated based on the precompiled reports. We hope that this resource will serve as a useful complement to the existing public resources and as a good starting point for researchers and physicians interested in EC genetics.

\section{Availability and requirements}

DDEC is freely accessible to academic and non-profit users at http://apps.sanbi.ac.za/ddec/.

\section{Competing interests}

Vladimir B. Bajic and Aleksandar Radovanovic are partners in the OrionCell company whose product, Dragon Exploration System (DES), has been used in creation of DDEC precompiled reports. Other authors declare no conflict of interest.

\section{Authors' contributions}

$\mathrm{ME}, \mathrm{MK}$ and VBB conceptualized the study, analyzed data and wrote the manuscript. AR, US, SVS, SS and AC developed the database. AR and VBB developed the DES system. All authors read and approved the final manuscript.

\section{Additional material}

\section{Additional file 1}

The implication of esophageal and ovarian cancer genes in KEGG pathways. This additional file is subdivided into three worksheets that list the genes common to EC and $O C$, genes unique to EC and genes unique to OC. Each worksheet further lists the gene name of each entry, the associated Entrez Gene ID and KEGG pathways.

Click here for file

[http://www.biomedcentral.com/content/supplementary/14712407-9-219-S1.xls]

\section{Acknowledgements}

ME was partly supported with a Scarce Skills Scholarship from the National Research Fund, South Africa; ME and VBB were partly supported by the National Research Foundation grant (61070); SRS, AC and VBB were supported partly by the DST/NRF Research Chair grant (6475I); VBB was partly supported by the National Research Foundation grant (62302). AR, US, SS and VBB were partly supported by the National Bioinformatics Network grants; MK has been supported by the postdoctoral fellowship from the Claude Leon Foundation, South Africa.

\section{References}

I. Stoner GD, Rustgi AK: Biology of the esophageal squamous cell carcinoma. Gastrointest Cancers Biol Diagn 1995, 8: 141-146.

2. WHO: The World Health Report 1997 - conquering suffering, enriching humanity. World Health Forum 1997, I 8:248-260.

3. Reed CE: Surgical management of esophageal carcinoma. Oncologist 1999, 4:95-105.

4. De LL, Curia MC, Aceto GM, Toracchio S, Colucci G, Russo A, Mariani-Constantini R, Cama A: Analysis of extended genomic rearrangements in oncological research. Ann Oncol 2007, 18 Suppl 6:vil73-vil78.

5. Gilbert N, Gilchrist S, Bickmore WA: Chromatin organization in the mammalian nucleus. Int Rev Cytol 2005, 242:283-336.

6. Cremer T, Cremer C: Chromosome territories, nuclear architecture and gene regulation in mammalian cells. Nat Rev Genet 2001, 2:292-301.

7. Kato K, Yamashita R, Matoba R, Monden M, Noguchi S, Takagi T, Nakai K: Cancer gene expression database (CGED): a database for gene expression profiling with accompanying clinical information of human cancer tissues. Nucleic Acids Res 2005, 33:D533-D536.

8. Thiemann KM, Frost MH, Thompson RA: A multifaceted educational approach to increasing awareness and use of physician data query (PDQ). J Cancer Educ 1999, I4:78-82.

9. Rhodes DR, Yu J, Shanker K, Deshpande N, Varambally R, Ghosh D, Barrette T, Pandey A, Chinnaiyan AM: ONCOMINE: a cancer microarray database and integrated data-mining platform. Neoplasia 2004, 6: I-6.

10. Eyre TA, Ducluzeau F, Sneddon TP, Povey S, Bruford EA, Lush MJ: The HUGO Gene Nomenclature Database, 2006 updates. Nucleic Acids Res 2006, 34:D3 I9-D32I.

II. Safran M, Solomon I, Shmueli O, Lapidot M, Shen-Orr S, Adato A, Ben-Dor U, Esterman N, Rosen N, Peter I, Olender T, Chalifa-Caspi V, Lancet D: GeneCards 2002: towards a complete, object-oriented, human gene compendium. Bioinformatics 2002, 18: I542-1543.

12. Kulikova T, Akhtar R, Aldebert P, Althorpe N, Andersson M, Baldwin A, Bates K, Bhattacharyya S, Bower L, Browne P: EMBL Nucleotide Sequence Database in 2006. Nucleic Acids Res 2007, 35:DI6-D20.

13. Flicek P, Aken BL, Beal K, Ballester B, Caccamo M, Chen Y, Clarke L, Coates G, Cunningham F, Cutts T: Ensembl 2008. Nucleic Acids Res 2008, 36:D707-D7I4.

14. Pruitt KD, Tatusova T, Maglott DR: NCBI reference sequences (RefSeq): a curated non-redundant sequence database of genomes, transcripts and proteins. Nucleic Acids Res 2007, 35:D6I-D65.

15. Benson DA, Karsch-Mizrachi I, Lipman DJ, Ostell J, Sayers EW: GenBank. Nucleic Acids Res 2009, 37:D26-D31.

16. Wheeler DL, Barrett T, Benson DA, Bryant SH, Canese K, Chetvernin V, Church DM, Dicuccio M, Edgar R, Federhen S: Database resources of the National Center for Biotechnology Information. Nucleic Acids Res 2008, 36:DI3-D2I.

17. UniProt Consortium: The universal protein resource (UniProt). Nucleic Acids Res 2008, 36:D I 90-DI 95.

18. Gasteiger E, Jung E, Bairoch A: SWISS-PROT: connecting biomolecular knowledge via a protein database. Curr Issues Mol Biol 200I, 3:47-55.

19. Berman H, Henrick K, Nakamura H, Markley JL: The worldwide Protein Data Bank (wwPDB): ensuring a single, uniform archive of PDB data. Nucleic Acids Res 2007, 35:D30I-D303.

20. Alibes A, Yankilevich $P$, Canada A, az-Uriarte R: ID converter and IDClight: conversion and annotation of gene and protein IDs. BMC Bioinformatics 2007, 8:9. 
21. Khatri P, Desai V, Tarca AL, Sellamuthu S, Wildman DE, Romero R, Draghici S: New Onto-Tools: Promoter-Express, nsSNPCounter and Onto-Translate. Nucleic Acids Res 2006, 34:W626-W63I.

22. Ashburner M, Ball CA, Blake JA, Botstein D, Butler H, Cherry JM, Davis AP, Dolinski K, Dwight SS, Eppig JT: Gene ontology: tool for the unification of biology. The Gene Ontology Consortium. Nat Genet 2000, 25:25-29.

23. Kelso J, Visagie J, Theiler G, Christoffels A, Bardien S, Smedley D, Otgaar D, Greyling G, Jongeneel CV, McCarthy MI: eVOC: a controlled vocabulary for unifying gene expression data. Genome Res 2003, 13:1222-1230.

24. Vastrik I, D'Eustachio P, Schmidt E, Gopinath G, Croft D, de Bono B, Gillespie M, Jassal B, Lewis S, Matthews L: Reactome: a knowledge base of biologic pathways and processes. Genome Biol 2007, 8:R39.

25. Carninci P, Sandelin A, Lenhard B, Katayama S, Shimokawa K, Ponjavic J, Semple CA, Taylor MS, Engstrom PG, Frith MC: Genome-wide analysis of mammalian promoter architecture and evolution. Nat Genet 2006, 38:626-635.

26. Aerts S, Van LP, Thijs G, Mayer H, de MR, Moreau Y, De Moor B: TOUCAN 2: the all-inclusive open source workbench for regulatory sequence analysis. Nucleic Acids Res 2005, 33:W393-W396.

27. Wingender E, Chen X, Fricke E, Geffers R, Hehl R, Liebich I, Krull M, Matys $\mathrm{V}$, Michael $\mathrm{H}$, Ohnhauser $\mathrm{R}$ : The TRANSFAC system on gene expression regulation. Nucleic Acids Res 200I, 29:28I-283.

28. Matys V, Kel-Margoulis OV, Fricke E, Liebich I, Land S, Barre-Dirrie A, Reuter I, Chekmenev B, Krull M, Hornischer K: TRANSFAC and its module TRANSCompel: transcriptional gene regulation in eukaryotes. Nucleic Acids Res 2006, 34:DI08-DI I0.

29. Kel AE, Gossling E, Reuter I, Cheremushkin E, Kel-Margoulis OV, Wingender E: MATCH: A tool for searching transcription factor binding sites in DNA sequences. Nucleic Acids Res 2003, 31:3576-3579.

30. Sagar S, Kaur M, Dawe A, Seshadri SV, Christoffels A, Schaefer U, Radovanovic A, Bajic VB: DDESC: Dragon database for exploration of sodium channels in human. BMC Genomics 2008, 9:622.

31. Pan H, Zuo L, Choudhary V, Zhang Z, Leow SH, Chong FT, Huang Y, Ong VW, Mohanty B, Tan SL: Dragon TF Association Miner: a system for exploring transcription factor associations through text-mining. Nucleic Acids Res 2004, 32:W230-W234.

32. Bajic VB, Veronika M, Veladandi PS, Meka A, Heng MW, Rajaraman K, Pan H, Swarup S: Dragon Plant Biology Explorer. A text-mining tool for integrating associations between genetic and biochemical entities with genome annotation and biochemical terms lists. Plant Physiol 2005, | 38: 19|4-1925.

33. Kaur M, Radovanovic A, Essack M, Schaefer U, Maqungo M, Kibler T, Schmeier S, Christoffels A, Narasimhan K, Choolani M, Bajic VB: Database for exploration of functional context of genes implicated in ovarian cancer. Nucleic Acids Res 2009, 37:D820-D823.

34. Smyth GK, Yang YH, Speed T: Statistical issues in cDNA microarray data analysis. Methods Mol Biol 2003, 224: I I I-I36.

35. Pritchard CC, Hsu L, Delrow J, Nelson PS: Project normal: defining normal variance in mouse gene expression. Proc Natl Acad Sci USA 200I, 98:13266-1327|.

36. Dennis G Jr, Sherman BT, Hosack DA, Yang J, Gao W, Lane HC, Lempicki RC: DAVID: Database for Annotation, Visualization, and Integrated Discovery. Genome Biol 2003, 4:3.

37. Goto S, Bono H, Ogata H, Fujibuchi W, Nishioka T, Sato K, Kanehisa $M$ : Organizing and computing metabolic pathway datain terms of binary relations. Pac Symp Biocomput 1997: 175-186.

\section{Pre-publication history}

The pre-publication history for this paper can be accessed here:

http://www.biomedcentral.com/1471-2407/9/219/pre pub
Publish with Biomed Central and every scientist can read your work free of charge

"BioMed Central will be the most significant development for disseminating the results of biomedical research in our lifetime. "

Sir Paul Nurse, Cancer Research UK

Your research papers will be:

- available free of charge to the entire biomedical community

- peer reviewed and published immediately upon acceptance

- cited in PubMed and archived on PubMed Central

- yours - you keep the copyright

Submit your manuscript here:

http://www.biomedcentral.com/info/publishing_adv.asp
BioMedcentral 